P\&A Año 2, N. ${ }^{\circ} 3$

julio-diciembre 2017

pp. [85]-98

\title{
Resumen
}

Para el diseño instruccional de una unidad del curso Ambientación y Color que se dicta en la carrera de Arquitectura de Interiores de la Universidad de Ciencias y Artes de América Latina, se toma como fundamento la teoría de aprendizaje de Skinner, ya que mediante ejercicios prácticos y precisos, y su pronta revisión, permite el aprendizaje al ir avanzando de un nivel al siguiente de manera gradual. Así, al ser esta una unidad que se basa no sólo en conocimientos concretos, sino también en las experiencias, emociones y factores sociales de los estudiantes recurrimos a la teoría de Vygotsky, la cual permite al estudiante potenciar sus propias habilidades para así obtener nuevos conocimientos de manera integral.

Palabras clave: Color, espacio, composición cromática, comunicación

\section{Ambientación \& Color: \\ Estrategias de Enseñanza y Aprendizaje en las asignaturas de expresión gráfica arquitectónica*}

\author{
Atmosphere \& Color \\ Strategies for Teaching and Learning in the subjects of architectural graphic \\ expression
}

Arq. Ana Cecilia Vega Reyes**

Arq. José Antonio Núñez Romero***

Recibido: 13 de abril de 2017

Aceptado: 20 de junio de 2017

\begin{abstract}
For the instructional design of a unit of the course Atmosphere and Color that runs at the career of architecture of interiors of the UCAL University, it is taken as a basis the theory of learning of Skinner, allowing precise and practical exercises and their prompt review learning to advance from one level to the next in a phased manner. As well as this is a unit that relies not only on specific expertise but also the experiences, emotions and social factors of the students, we turn to the theory of Vygotsky, which enables students to enhance their own abilities to obtain new knowledge in a comprehensive way.
\end{abstract}

Keywords: Color, space, chromatic composition, chromatic expressivity.

${ }^{*}$ Este artículo es el resultado de las implementaciones pedagógicas desarrolladas por los autores durante el dictado de una unidad de aprendizaje del curso de Ambientación y Color en la Carrera de Arquitectura de Interiores de la Universidad de Ciencias y Artes de América Latina.

** Arquitecta por la Universidad Ricardo Palma, Maestrista en Docencia Universitaria y Gestión Educativa por la Universidad Técnológca del Perú. Con más de 10 años de experiencia docente en la URP-FAU y actualmente se desempeña como docente en la Universidad de Lima y Universidad de Ciencias y Artes de América Latina.

*** Arquitecto por la Universidad Ricardo Palma, Maestrista en Docencia Universitaria y Gestión Educativa por la UTP, tiene un Diplomado en Docencia Universitaria por la Universidad de San Martín de Porres, con más de 10 años de experiencia docente en la URP-FAU, Universidad Peruana de Ciencias Aplicadas y UCAL 
Esta metodología pretende ayudar al alumnado a organizar su pensamiento favoreciendo en ellos la reflexión, la crítica, la elaboración de hipótesis y la tarea investigadora a través de un proceso en el que cada uno asume la responsabilidad de su aprendizaje, aplicando sus conocimientos y habilidades a proyectos reales. Se favorece, por tanto, un aprendizaje orientado a la acción en el que se integran varias áreas o materias: los estudiantes ponen en juego un conjunto amplio de conocimientos, habilidades o destrezas y actitudes personales, es decir, los elementos que integran las distintas competen-

cias.

Ministerio de Educación, Cultura y Deporte, 2015

\section{Introducción}

Ante los tiempos de cambios, los sistemas pedagógicos también deben transformarse a fin de afrontar grandes retos como garantizar el desarrollo personal, con respeto a la diversidad y con equidad, y participar de una sociedad cambiante teniendo la capacidad de vivir en comunidad.

Se hace necesario el cambio en la formación de las personas, por lo tanto, el cambio de los procesos de enseñanza-aprendizaje y la labor docente, así como la reformulación de tareas pedagógicas que busquen el desarrollo de competencias entendidas como:

Es importante ligar el aprendizaje a problemas prácticos, del mundo real, de manera que se valoren los conocimientos por el uso que se les puedan dar.

El entorno actual exige el cambio hacia la aplicación de metodologías de trabajo que sean más flexibles, que inviten a la reflexión, que favorezcan el aprendizaje con sistemas de trabajo colaborativo estableciendo ayuda entre iguales.

Es en este contexto donde se sitúa el presente artículo que expone y describe la elaboración de una unidad de aprendizaje del curso de Ambientación y Color de la carrera de Arquitectura de Interiores de la Universidad de Artes y Ciencias de Latinoamérica (UCAL). Cada sesión de la unidad es presentada de manera gráfica tal cual es mostrada y preparada junto al estudiante para mantener una sintonía de acuerdo con la naturaleza del curso. La asignatura es de carácter obligatorio para los alumnos del segundo ciclo de la carrera de Arquitectura de interiores (AI) y electivo para los estudiantes de Diseño Gráfico y Comunicaciones.

La incidencia de los gráficos y colores en un documento, en otras ocasiones demasiado solemnes para un curso sobre el uso e importancia del color, permitió observar a los autores del artículo, el cambio de mentalidad y la motivación ocurrida sobre el alumnado y sus aprendizajes significativos, así como la pertinencia del uso de una metodología de enseñanza que tienda más a lo sensorial. Este fue el motivo por el cual el desarrollo de la asignatura cambió radicalmente del solo enunciado del encargo cognitivo a la búsqueda de competencias conceptuales, procedimentales y actitudinales activadas por gráficos descriptivos, símbolos llamativos y coloridos expresados en todo el material pedagógico.

Para cada sesión de clase se han considerado la aplicación de dos teorías de aprendizaje: La teoría de Burrhus Frederic Skinner y su "condicionamiento operante" $(1938)^{1}$ (entendiendo el condicionamiento al uso repetido del color como estímulo) y Lev Vygotsky con la "teoría sociocultural del desarrollo cognitivo" (2012) (la cual nos permite entender el uso cultural del color por parte de cada alumno y su entorno).

Ha sido de interés fundamentar el estudio y análisis concienzudo de las teorías de aprendizaje por parte de los autores. La aplicación de estas en las metodologías del curso de ambientación y color permite el desarrollo de competencias y capacidades que van a facultar al estudiante desarrollarse de la mejor manera a futuro. Es así como se busca el desarrollo integral de la persona, en lo cognitivo, afectivo, procedimental y social. Se fomenta el proceso de adquisición de macro-habilidades para ser útil a la so-

1 En 1938, Frederic Skinner acuñó el término "acondicionamiento operante". 
ciedad y la realización personal, valorando los procesos de interculturación.

Al finalizar el curso, cada alumno logra aplicar el color como elemento de significación y relevancia en el diseño de un estand de exhibición de una marca determinada en una feria internacional, presentando para este fin un portafolio técnico, una maqueta coloreada a escala $1 / 25$ y un panel de sustentación.

El nombre de la unidad a desarrollar "Mi mundo a color", refuerza la pertinencia de la metodología aplicada, permitiendo observar la destreza del alumno en la utilización del collage en una perspectiva en armonía y en contraste como herramientas de composición cromática. La unidad presentada en el artículo comprende 5 sesiones de aprendizaje de dos horas por sesión.

\section{La importancia del color en el entorno de las personas}

El color es expresión, es energía radiante y es comunicación. Es de vital importancia conocer los efectos que el color ejerce sobre los diferentes espacios y por ende sobre las personas que los habitan y consumen. El color puede llegar a transformar los espacios en lugares donde se es muy agradable permanecer o por el contrario espacios que se vuelven incómodos y poco confortables.

Es materia de este curso incentivar en los alumnos el interés por descubrir la importancia del uso del color, que sean ellos mismos los que por medio de ejercicios exploratorios descubran las posibilidades de las diferentes paletas cromáticas para luego aplicarlas logrando el efecto deseado y que este no sea obtenido por casualidad.

Cada ejercicio planteado está diseñado para que los participantes del curso exploren de manera práctica la teoría del color y así descubrir que es necesaria su valoración. Se incentiva el uso de herramientas que servirán para poder aplicar el color con asertividad y entusiasmo, expresando las cualidades emocionales y formales deseadas.

\section{Teoría del color y la enseñanza de su construcción}

Los ejercicios planteados y expuestos en el artículo son resultado de revisión de dos importantes investigadores en la materia. El primero, Johann Wolfgang von Goethe, quien en su libro Zur Farbenlehre (Teoría de los colores) de 1810, expone sobre la percepción, estética y psicología del color. Se opuso a la teoría de Newton ya que no veía al color solamente como un fenómeno de la física y la matemática, sino que también era importante el estudio del sentido de la vista y de cómo el cerebro procesa e interpreta el color (percepción). Es aquí donde radica la importancia de su investigación: La trascendencia simbólica de los colores y cómo estos responden a una experiencia personal.

Además, Goethe estudió las reacciones humanas a las diferentes agrupaciones de colores y marcó el inicio del tratado de la sicología moderna del color.

Ya que, en el paso de la experiencia al juicio, del conocimiento a la aplicación, es donde, como en un desfiladero, todos los enemigos interiores del hombre están al acecho: la imaginación [...], la impaciencia, la precipitación, la autocomplacencia, la testarudez, la mentalidad, las opiniones preconcebidas, la pereza, la ligereza, la volubilidad. (Goethe, 1790/1997, p. 157)

El segundo investigador citado es Johannes Itten (1888-1967), con su libro Arte del Color, quien maneja la enseñanza de la construcción de los colores mediante las leyes fundamentales de los efectos cromáticos que se derivan de la intuición.

Revisando a Goethe e Itten para el estudio del color, se han propuesto ejercicios detallados para lograr el aprendizaje. Los alumnos perciben los objetos, los espacios y las sensaciones y de alguna manera están intuyendo qué atmósfera se genera tras su aplicación y cómo manipular este significado en beneficio de un mensaje transmitido por un espacio arquitectónico, una silla, un florero o cualquier objeto que lo componga (ver figura 1). 


\section{Teorías de aprendizaje: Skinner y Vygotsky}

Luego de entender la trascendencia de la teoría del color y su necesario dominio por parte de los alumnos, para la manipulación del espacio en beneficio del mensaje arquitectónico, se planteó incorporar en la metodología actual del curso dos teorías claves del aprendizaje moderno que complementen el correcto desarrollo de la unidad.

La primera referencia es la incorporación de la teoría del condicionamiento operante de Frederick Skinner (USA, 1904-1960), psicólogo norteamericano y profesor de la Universidad de Harvard en 1948, año en el cual escribió su novela de ciencia ficción Walden Dos, donde refuerza sus investigaciones sobre el conductismo, esta novela describe un mundo ideal centrado en la utilización de la ciencia para resolver la vida de los individuos sin envidias ni maldades en busca de la felicidad de su comunidad. En su momento este documento tuvo muchos detractores que vieron en esta novela-ensayo-ficción un manifiesto opresor de la libertad del individuo, a pesar de ello el interés de Skinner sería más llevado a terminar con la desigualdad y jus-

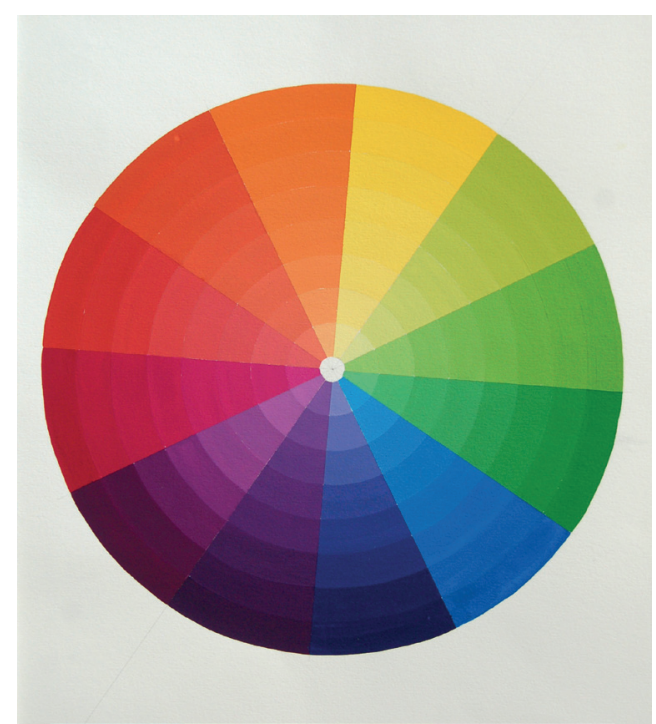

Figura 1. Círculo cromático. Trabajado en clase relacionando la teoría de Goethe y la de Itten tificar su teoría del condicionamiento operante como método de aprendizaje efectivo y significativo. El condicionamiento operante como una característica del conductismo, permite al estudiante un aprendizaje significativo mediante la realización de una acción que está seguida de un estímulo deseable, el cual permite a la persona realizar la acción nuevamente, alojando así de manera permanente el conocimiento, habilidad o actitud en su mente y posterior comportamiento.

Esta teoría se trasladó a la metodología aplicada en el curso de manera puntual y por unidades de aprendizaje su integración se dio de manera gradual, permitiendo entender que para cada estímulo sensorial hay una respuesta condicionada; esta respuesta autoriza al alumno ya estimulado a trabajar realizando "pequeños pasos" que fomenten una respuesta activa y que otorguen una rápida comprobación y feedback para pasar al siguiente paso y así sucesivamente ir comprobando los conceptos de clase, todo esto iniciado por un problema-estímulo pensado para la realización de una acción y creación de una conducta específica, es decir, a cada estímulo le corresponde una respuesta significativa. Esta pedagogía permite al alumno encontrar su propio ritmo de trabajo ante pre-existencias específicas que le brinda el color mediante ensayos sucesivos en pequeñas unidades de tiempo.

Ejemplo de la aplicación en el curso:

-Se plantea un problema-estímulo: ¿qué color tendrá mi espacio favorito?

-Etapa de desarrollo: Cada estudiante expresa su propuesta de forma gráfica, utilizando el color que refleje mejor su parecer.

-Se llega a una respuesta rápida: a través de la crítica colectiva para "descubrir" las diferentes posibilidades en la aplicación del color. Valorar lo concreto y lo subjetivo de cada propuesta, así como poner en valor y enriquecer el conocimiento.

-El replanteo: luego de la crítica el estudiante debe mejorar su propuesta, con un mejor sustento aplicativo. 